\title{
CONSUMO DE ALIMENTOS IN NATURA POR PACIENTES PORTADORES DEDOENÇAS CRÔNICAS NÃO TRANSMISSÍVEIS DA CLÍNICA DE SAÚDE DA ESTÁCIO DO RECIFE
}

\author{
IN NATURA FOOD CONSUMPTION BY PATIENTS WITH NON-TRANSMISSIBLE \\ CHRONIC DISEASES OF THE ESTÁCIO DO RECIFE HEALTH CLINIC
}

\author{
Bruna Teodoro dos Santos ${ }^{1}$ \\ Hellba Karts Maria e Silva ${ }^{2}$ \\ Joyce Oliveira de CarvalhoBarros ${ }^{3}$ \\ Steffany Kelly Pontes Pires ${ }^{4}$
}

\begin{abstract}
RESUMO: O consumo excessivo dos industrializados é uma das principais causas de problemas de saúde. $\mathrm{O}$ uso desses produtos é estimulado pela praticidade, custo baixo, grandes porções e pelo marketing das embalagens e das mídias. Segundo o Guia Alimentar da População Brasileira preconiza que o consumo dos ultra processados e minimamente processados estão afetando de modo desfavorável a cultura, a vida social e o meio ambiente (OMS,2014). O primeiro passo para uma alimentação saudável é fazer uso de alimentos in natura ou minimamente processados a base da alimentação. Por isso é importante investigar o consumo de alimentos in natura por indivíduos portadores de DCNT. Objetivo: investigar o consumo de alimentos in natura em pacientes portadores de doenças crônicas não transmissíveis da clínica de saúde da Estácio do Recife-PE. Método: trata-se de um estudo transversal retrospectivo, desenvolvidos com pacientes da clínica de saúde da Estácio do Recife, onde foram coletadas as informações sobre o consumo de alimentos in natura por estes pacientes através das fichas de atendimento da clínica. Resultados: Foram avaliados 20 pacientes de ambos os sexos, na faixa de idade entre 30 a 50 anos, $75 \%(n=15)$ eram mulheres e $25 \%(=5)$ eram homens. Em relação às variáveis, pôde-se analisar que $65 \%(=13)$ não eram portadores de diabetes, enquanto $35 \%(n=7)$ possuíam a doença. $\mathrm{Na}$ variável de HAS foi observado que $65 \%(n=13)$ eram portadores da HAS, enquanto $35 \%(n=7)$ não possuíam a doença. Sobre câncer e obesidade houve resultados com baixa prevalência nos pacientes avaliados sendo $10 \%(n=2)$ possuem e $90 \%(n=18)$ não são portadores da doença, enquanto $5 \%(n=1)$ possuem e $95 \%(n=19)$ não são portadores respectivamente. Nas variáveis do consumo de alimentos in natura, foi observado que $50 \%(\mathrm{n}=10)$ dos pacientes consomem frequentemente esse tipo de alimento e os outros 50\% $(\mathrm{N}=10)$ consomem pouco. Conclusão:
\end{abstract}

\footnotetext{
I Estudante de Nutrição-Estácio: E-mail: bruninhalayne@hotmail.com

${ }^{2}$ Estudante de Nutrição-Estácio: E-mail: hellbakarts@gmail.com

${ }^{3}$ Estudante de Nutrição-Estácio: E-mail:oliverjoyceı@@gmail.com

${ }^{4}$ Docente do curso de Nutrição-Estácio: E-mail: steffanypontes.nutricionista@outlook.com
} 
Através da pesquisa realizada foi possível identificar que o consumo frequente de alimentos in natura por parte dos portadores de DCNT equivale apenas à metade da amostra. Revelando que apesar deo consumo de alimentos in natura e minimamente processados ser o primeiro passo para uma alimentação saudável, o consumo de frutas, verduras e legumes ainda é relativamente baixo. Por se tratar de um estudo com uma pequena quantidade de participantes, baseado em seus dados, é necessária a realização de novos estudos que identifiquem a relação entre o consumo de alimentos in natura e portadores de DCNT.

Palavras chaves: Alimentos naturais. doenças crônicas não transmissíveis. consumo alimentar. fatores de risco.

ABSTRACT: Excessive consumption by the industrialized is one of the main causes of health problems. The use of these products is stimulated by practicality, low cost, large portions and the marketing of packaging and media. According to the Food Guide for the Brazilian Population, it is recommended that the ultra-processed and minimally processed consumption foods is having an adverse effect on culture, social life and the environment (WHO, 2014). The first step towards healthy eating is to make use of fresh or minimally processed foods based on food. That is why it is important to investigate the consumption of fresh food by individuals with CNCD. Objective: To investigate the consumption of fresh food in patients with chronicnon-communicable diseases at the Estácio do Recife-PE health clinic. Method: This is a retrospective cross-sectional study, developed with patients from the Estácio do Recife health clinic, where the information oconsumption of fresh foods by these patients was collected through the clinic's attendance sheets. Results: Twenty patients of both sexes were evaluated, aged between 30 and 50 years, $75 \%(n=15)$ were women and $25 \%$ (= 5) were men. Regarding the variables, it was possible to analyze that $65 \%(=13)$ did not have diabetes, while $35 \%(n=7)$ had the disease. In the SAH variable, it was observed that $65 \%(n=13)$ had SAH, while $35 \%(n=7)$ did not have the disease. Regarding cancer and obesity, there were results with low prevalence in the patients evaluated, I0\% $(n=2)$ having and $90 \%(n=18)$ not having the disease, while 5\% $(n=1)$ have and 95\% $(n=19)$ are not carriers respectively. In the consumption variables of fresh food, it was observed that $50 \%(\mathrm{n}=10)$ of patients frequently consume this type of food and the other $50 \%(\mathrm{~N}=10)$ consume little. Conclusion: Through the research carried out it was possible to identify that the consumption frequent of fresh food by the patients with CNCD is only equivalent to half of the sample. Revealing that although the fresh and minimally processed foods consumption is the first step towards a healthy diet, the fruits and vegetables consumption is still relatively low. As this is astudy with a small number of participants, based on their data, it is necessary to carry out new studies that identify the relationship between the consumption of fresh foods and those with CNCD.

Keywords: Natural foods. chronic non-communicable diseases. food consumption. risk factors

\section{INTRODUÇÃO}

As doenças crônicas não transmissíveis (DCNT) constituem sério problema de saúde pública, tanto nos países ricos quanto nos de média e baixa renda Caracterizam-se 
por ter umaetiologia incerta, múltiplos fatores de risco, longos períodos de latência, curso prolongado, origem não infecciosa e por estarem associadas a deficiências e incapacidades funcionais. Dentre as principais estão as doenças cardiovasculares, os cânceres, as doenças respiratórias crônicas e o diabetes mellitus (BAUMGARTL C. et al, 20ı6).

Essas patologias são consideradas sérios problemas de Saúde Pública. Desenvolvendo-se ao longo da vida, elas produzem graves complicações, de forte impacto na morbimortalidade e na qualidade de vida dos indivíduos afetados, maior risco de morte prematura e efeitos econômicos adversos para as famílias, comunidades e sociedade geral. Segundo dados da Organização Mundial da Saúde (OMS), esse grupo de doençasrepresentou 74,0\% das mortes no Brasil em 2016 (BARROS C. et al,2019 ) .

As DCNT compartilham diversos fatores de risco, como hereditariedade, raça, sexo, tabagismo, consumo excessivo de álcool, dislipidemias, consumo insuficiente de frutas, legumes e verduras e sedentarismo. A inatividade física e o excesso de peso são responsáveis, respectivamente, por 3,2 e 2,8 milhões de mortes/ano (FILHO B. et al,2019).

O consumo excessivo dos industrializados é uma das principais causas de problemas de saúde. Com seus altos níveis de gorduras trans e saturadas, alto teor de sal e açúcar que trazem consigo um sabor viciante. $\mathrm{O}$ uso desses produtos é estimulado pela praticidade, custo baixo, grandes porções e pelo marketing das embalagens e das mídias. Segundo o Guia Alimentar da População Brasileira preconiza que o consumo dos ultra processados e minimamente processados estão afetando de modo desfavorável a cultura, a vida social e o meio ambiente (OMS,2014).

Medidas de ação global estão sendo tomadas pela OMS a fim de reduzir $25 \%$ das mortes prematuras por DCNTs até 2020. Neste plano de ação estão nove metas globais voluntárias que abordam principais fatores de risco para DCNT, incluindo uso de tabaco, ingestão de sal, inatividade física, pressão alta e uso nocivo de álcool (OMS,2014).

O primeiro passo para uma alimentação saudável é fazer uso de alimentos in natura ou minimamente processados a base da alimentação. Alimentos esses, ricos em vitaminas e minerais, fibras, flavonoides e antioxidantes que, associados a atividades físicas podem ser benéficos na prevenção de doenças como, cardiovasculares, diabetes, síndrome metabólica, câncer, entre outros. Por isso é importante investigar o consumo desses 
alimentos por indivíduos portadores de DCNT. 


\section{METODOLOGIA}

Trata-se de um estudo transversal retrospectivo, desenvolvido com pacientes portadores de doenças crônicas não transmissíveis (DCNT) da clínica de saúde que fica localizada no Centro Universitário Estácio do Recife.

Foram incluídos na pesquisa, todos os indivíduos na faixa etária de 30 a 50 anos que foram atendidos entre o período de maio de 2019 a setembro de 2019, que apresentaram diabetes, hipertensão, câncer, doenças respiratórias crônicas ou obesidade.

Para a realização da pesquisa, os dados foram coletados após submissão e aprovação ao comitê de ética da Estácio do Recife sob o número de CAAE: 28875320.2.00oo.5640 de acordo com a resolução no $466 / 12$ do Conselho Nacional de saúde, foi enviado um termo de dispensa do TCLE, pois, as informações obtidas foram de materiais secundários analisados através das fichas de atendimento da clínica de saúde da Estácio do Recife e não houve contato direto com os pacientes.

Foram avaliados o consumo de alimentos in natura pelos pacientes portadores de DCNT, especificamente, as frutas, verduras e legumes, o modo como foi avaliado foi se eles não consomem, consomem pouco ou consomem frequentemente os alimentos citados acima.

Para investigação do consumo desses alimentos, foi desenvolvido um formulário próprio, contendo o nome completo dos pacientes, a idade e sexo, a patologia de base, hipertensão, diabetes, câncer, doença respiratória crônica ou obesidade, e a frequência de consumo dos alimentos. $\mathrm{O}$ mesmo encontra - se no apêndice $\mathrm{I}$.

A análise dos dados foram realizadas com o auxílio do programa Microsoft Excel 2010 e serão apresentas em forma de tabela dentro do texto.

\section{DESENVOLVIMENTO}

De acordo com a OMS (2016) as doenças crônicas não transmissíveis são as principaiscausas de óbitos no mundo e seus números de casos vêm crescendo ao longo dos anos. Com as novas tecnologias, inclusive de alimentos, o consumo dos alimentos industrializados é muito alto pela sua praticidade, economia, maior tempo de prateleira, sabores concentrados e grandes porções que atraem as pessoas ao consumo.

Dentre as causas que contribuem para o desenvolvimento das DCNTs, a 
Organização Mundial da Saúde pontua os quatro principais fatores, sendo eles o fumo, o sedentarismo, o uso prejudicial de álcool e a má alimentação (DUNCAN, 2012).

O consumo preferencial dos alimentos processados e ultra-processados é um problemae impede que o indivíduo agregue a sua alimentação alimentos que contenham mais fibras, antioxidantes, vitaminas, minerais que são essenciais para a saúde. Atuando como reguladorese protetores contra radicais livres e dislipidemias (Matos et al., 2019).

O Guia Alimentar para a população Brasileira preconiza que a base da alimentação deve ser composta de alimentos in natura, que alimentos provenientes da natureza sem nenhuma alteração, ou minimamente processados, que são alimentos que sofrem alguma alteração como secagem, limpeza, moagem, retirada de partes não comestíveis ou indesejadas, pasteurização. Controlar o consumo de alimentos processados e evitar o consumo dos alimentos que tiveram adição de sal, açúcar ou passou por processamento de conservação, ultra-processados alimentos que contém aditivos ou passaram por processo muitas vezes de exclusividade industrial. (BRASIL, 2014b).

Um estudo realizado por Santos et al. (2016) verificou associação entre a compra de alimentos industrializados com a alta taxa de prevalência de DCNTs, relacionadas ao frágil conhecimento em educação e saúde da população em geral e também à interpretação de rótulos de alimentos.

Contudo, o objetivo do presente estudo foi avaliar o consumo de alimentos in natura por pacientes com doenças crônicas não transmissíveis usuários do ambulatório da clínica de saúde da Estácio do Recife, Pernambuco.

\section{RESULTADOS}

A amostra foi composta por 20 pacientes, variando entre homens e mulheres, na faixa etária entre 30 e 50 anos.

Analisando as características do estudo, houve predominância do sexo feminino, sendoele de $75 \%(n=15)$, e o sexo masculino totalizou $25 \%(n=5)$.

O Diabetes é caracterizada pela produção insuficiente ou má absorção de insulina, hormônio que regula a glicose no sangue e garante energia para o organismo. Em 2017, a Federação Internacional de Diabetes (International Diabetes Federation, IDF) estimou que $8,8 \%$ da população mundial com 20 a 79 anos de idade (424,9 milhões de pessoas) vivia com diabetes, sendo o Brasil o $4^{\mathrm{o}}$ país no mundo com o maior número de indivíduos (I2,5 
milhões) acometidos com essa doença. No estudo, foi observado que $35 \%(n=7)$ das pessoas analisadas possuem diabetes, sendo elas, todas as mulheres e nenhum homem, e $65 \%(n=13)$ não possuem a doença.

Hipertensão arterial (HA) é condição clínica multifatorial caracterizada por elevação sustentada dos níveis pressóricos $\geq 140 \mathrm{e} / \mathrm{ou} 90 \mathrm{mmHg}$. Segundo a diretriz Brasileira de Hipertensão (2016), no Brasil, a HÁ atinge 32,5\% (36 milhões) de indivíduos adultos e mais de 60\% dos idosos. No presente estudo, analisamos que $65 \%(n=13)$ dos indivíduos possuem Hipertensão, sendo 7 mulheres e os 5 homens que foram constados na pesquisa, e $35 \%(n=7)$ não possuem a doença.

Segundo o INCA, o câncer caracteriza-se pela perda do controle da divisão celular e pela capacidade de invadir outras estruturas orgânicas. Atualmente, câncer é o nome geral dado a um conjunto de mais de ioo doenças, que têm em comum o crescimento desordenado de células que tendem a invadir tecidos e órgãos vizinhos. E no estudo realizado, a prevalência do câncer foi baixa, obtendo Io\% $(\mathrm{n}=2)$ de indivíduos com a doença e 90\% (=I8) não apresentaram, sendo os que apresentaram, pessoas do sexo feminino.

A obesidade, integra o grupo de Doenças Crônicas Não-Transmissíveis, ela é caracterizada pelo acúmulo excessivo de gordura corporal em extensão tal, que acarreta prejuízos à saúde dos indivíduos e atualmente, é um problema de saúde pública mundial, tanto os países desenvolvidos como os em desenvolvimento apresentam elevação de sua prevalência. $\mathrm{Na}$ pesquisa foi constado apenas $5 \%(\mathrm{n}=\mathrm{I})$ do número de indivíduos que possuem a doença enquanto $95 \%(\mathrm{n}=19)$ não possuem.

$\mathrm{Na}$ pesquisa também foi avaliado o consumo de alimentos in natura por esses indivíduos portadores de doenças crônicas não transmissíveis. Sabendo que atualmente, o consumo de industrializados tem sido constantes na vida dos indivíduos, e sendo eles um fatorimportante na causa de várias doenças, dentre elas a DCNT, investigar o consumo dos alimentos in natura é importante, pois os mesmos possuem benefícios tanto na prevenção, como no tratamento dessas doenças (Martins; Faria, 2018). No presente estudo foi constatado que 50\% $(\mathrm{n}=10)$ dos indivíduos apresentados na pesquisa consomem esse tipo de alimento frequentemente, enquanto os outros $50 \%(\mathrm{n}=10)$ consomem pouco.

$\mathrm{Na}$ tabela I, estão descritos as variáveis demográficas, nutricionais e os resultados 
da pesquisa feita com os indivíduos atendidos na clínica de saúde do Centro Universitário Estácio da cidade do Recife-PE,2019.

Tabela I. Caracterização das variáveis demográficas e nutricionais de indivíduos atendidos em clínica de saúde da cidade do Recife-PE.

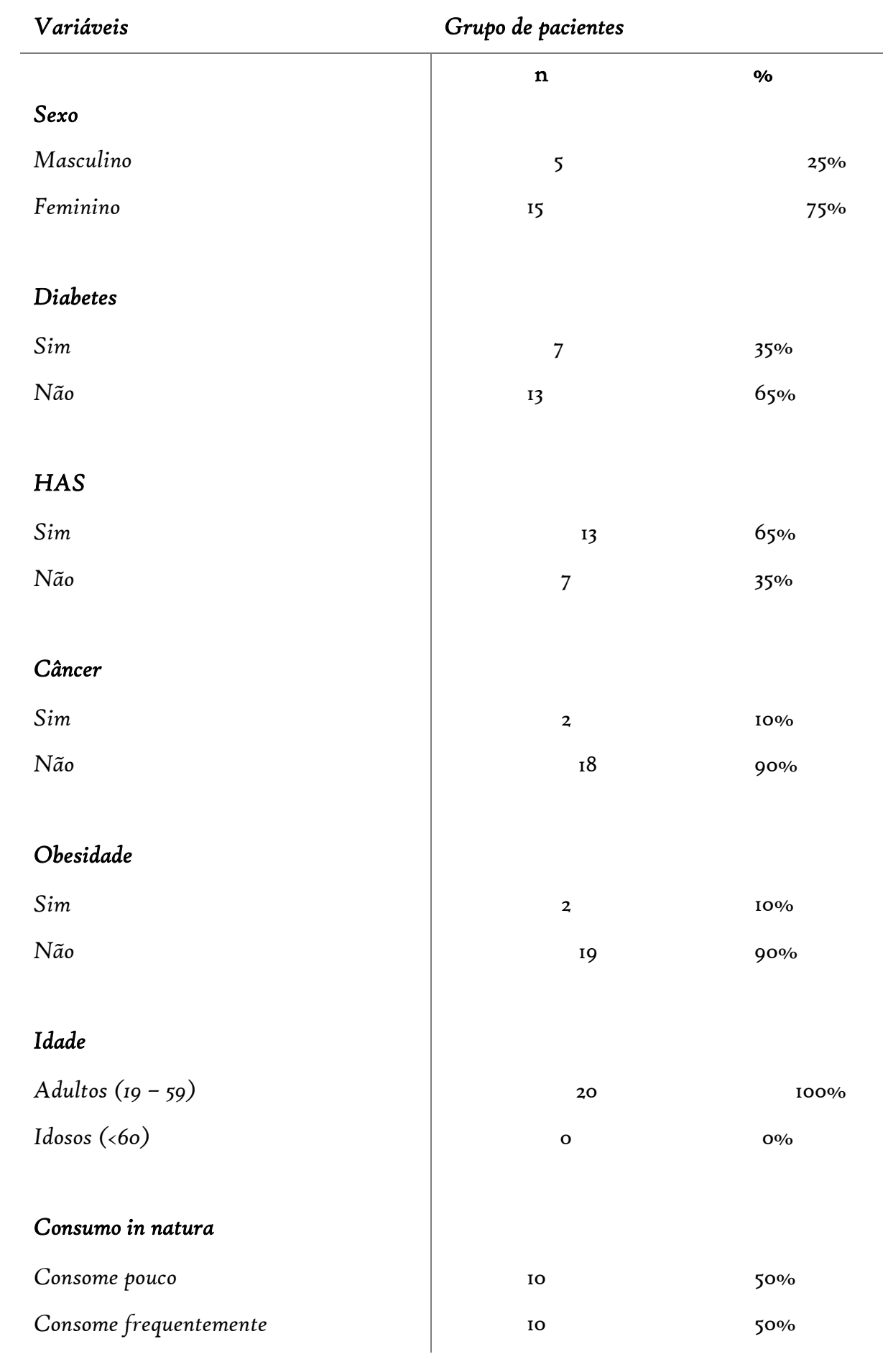


$0 \%$ 


\section{DISCUSSÃO}

As Doenças Crônicas Não Transmissíveis (DCNTs) são hoje uma das principais causas de mortes no Brasil e no mundo. Essas enfermidades acometeram grande parte da população nos últimos anos, afetando diretamente a qualidade de vida em razão de suas limitações e grandes custos econômicos (MALTA, 2014). A adoção de uma vida saudável e de hábitos alimentares adequados pode contribuir para uma boa qualidade de vida e para a prevenção e/ou redução das DCNTs. Conforme exposto anteriormente, a maioria dos fatores que causam essas doenças está relacionada a hábitos de vida inadequados. Assim, umaalimentação saudável é sinônimo de redução do risco de desenvolvimento de DCNTs (BRASIL, 2014b).

No estudo presente, ao comparar o estado clínico entre os sexos, verificou-se maior prevalência de DCNTs entre as mulheres (75\%). E foi observada uma frequência do consumo de alimentos in natura por metade da população estudada em ambos os sexos e estados nutricionais.

Ficou constatado que 50\% dos participantes apresentavam o consumo frequente de alimentos in natura em sua alimentação, mesmo assim apresentam alguma DCNT, este achado é semelhante ao estudo de Caetano, V.C.; et al.(2017) realizado no período entre abril de 2013 a abril de 2017, na cidade de Juiz de Fora, Minas Gerais, com 93 participantes, sendo $72,0 \%$ mulheres e a maioria dos participantes apresentavam obesidade e hipertensão arterial. Foi possível observar maior contribuição dos alimentos in natura na ingestão calórica, de macronutrientes e de fibras na alimentação dos indivíduos com excesso de peso avaliados no presente estudo. Sabemos, no entanto, que o consumo adequado de frutas, legumes e vegetais é um fator de porte. O que nos leva a concluir que muitos dos pacientes só adotam hábitos alimentares saudáveis após já possuírem alguma patologia.

Sobre as DCNTs, todos os avaliados possuem alguma dessas doenças, sendo a hipertensão a mais prevalente $(n=13), 65 \%$. Tal informação tem semelhante resultado com os dados de Neitzel, B.; et al.(2018) que avaliou 75 pessoas no posto de saúde municipal da cidade de Ipiranga (PR) e constatou que a maioria das pessoas que possuíam alguma DCNTs eram hipertensas $(n=24)$.

Pode-se observar, também, maior frequência no consumo de alimentos in natura por parte dos hipertensos, entrando em divergências com os resultados encontrados no 
estudo de Ferreira, R.C.; et al.(2019), realizado com 655 adultos, hipertensos, de ambos os sexos, estudados no período de 2013 a 2016, onde se identificou que os alimentos processados eultra processados faziam parte do consumo alimentar dos hipertensos investigados. Embora o consumo desses alimentos tenha sido inferior ao de alimentos in natura/minimamente processados, sabe-se que a alimentação desempenha papel importante nas DCV, constituindo fator de risco ou de proteção e que a exclusão desses alimentos da dieta é preconizada.

O Consumo de alimentos in natura é de suma importância para garantir a ingestão ideal de vitaminas, minerais, antioxidantes e fibras, a fim de prevenir e tratar as DCNTs.

Fica evidente a necessidade de atividades de educação nutricional, para estimular hábitos de vida saudável e melhorar a qualidade de vida desses indivíduos (Neitzel, 2018).

O Nutricionista é o um único profissional capacitado para prescrição de dietas e sua atuação é imprescindível para orientar o indivíduo sobre alimentação e hábitos de vida saudável.

Algumas limitações do estudo precisam ser consideradas: Se trata de um estudo retrospectivo, onde não houve contato direto com os pacientes e as informações obtidas foramde materiais secundários das fichas de atendimento da clínica de saúde da Estácio do Recife. Como também a quantidade de participantes na pesquisa, que por ser um valor pequeno, pois não se encaixavam nos critérios de inclusão, dificulta alguns tipos de análises, tendo em vista as circunstâncias presentes que estamos enfrentando não houve a possibilidade de coleta de mais dados para melhor conclusão do estudo.

\section{CONCLUSÃO}

Através da pesquisa realizada foi possível identificar que o consumo frequente de alimentos in natura por parte dos portadores de DCNT equivale apenas à metade da amostra. Revelando que apesar de o consumo de alimentos in natura e minimamente processados ser o primeiro passo para uma alimentação saudável, o consumo de frutas, verduras e legumes ainda é relativamente baixo.

Por se tratar de um estudo com uma pequena quantidade de participantes, baseado em seus dados, é necessária a realização de novos estudos que identifiquem a relação entre o consumo de alimentos in natura e portadores de DCNT.

Considerando a alta prevalência de DCNT no âmbito global e tendo por 
conhecimento que uma alimentação com base em alimentos in natura pode contribuir na prevenção, ajudar no controle dessas patologias, é de extrema importância a educação nutricional, pois possibilita intervenções preventivas e melhorias na qualidade de vida dos portadores. Incentivando e conscientizando a população sobre a importância da inclusão de alimentos in natura no seu dia a dia e como eles se mostram como fatores de proteção contra as DCNT.

\section{REFERÊNCIAS}

ABESO, Diretrizes Brasileiras de Obesidade. São Paulo, 2016, 4르 edição. Disponível em:https://abeso.org.br/wp-content/uploads/2019/12/Diretrizes-Download-DiretrizesBrasileiras- de-Obesidade-2016.pdf. Acesso em: 2 Jun. 2020.

BARROS, F.C. et al. Doenças crônicas não transmissíveis em profissionais de enfermagem de um hospital filantrópico no Sul do Brasil. Brasília: Epidemiologia e Serviços de Saúde vol.28 no.2. Disponível em: http://dx.doi.org/10.5123/si6794974201900o200ori.Acesso em: i Nov. 2019.

BAUMGARTEL,C. et al. Fatores de risco e proteção de doenças crônicas em adultos: estudo de base populacional em uma cidade de médio porte no sul do Brasil. Rio de Janeiro: Revista Brasileira de Medicina de Família e Comunidade. Disponível em: https://www.rbmfc.org.br/rbmfc/article/viewFile/1248/828. Acesso em:I Nov.2019.

CAETANO, C.V. et al. Consumo de alimentos processados e ultraprocessados em indivíduos adultos com excesso de peso. Juiz de Fora: HU Revista, v.43, n.3, p. 355-362, out/dez 2017. Disponível em: https://doi.org/ro.34019/1982-8047.2017.v43.2861. Acesso em: 03 Jun. 2020.

FILHO,B.M. et al. Doenças crônicas não transmissíveis e fatores associados em adultos numaárea urbana de pobreza do nordeste brasileiro. Rio de Janeiro: Ciência Saúde Coletiva vol.24 no.8. Disponível em: http://dx.doi.org/10.1590/1413-81232018248.30742017

. Acesso em: I Nov. 2019.

MARTINS, A.F.P; FARIA, C.R.L. Alimentos ultraprocessados: uma questão de saúde pública. Comunicação em Ciências da Saúde; 2018. Disponível em: http://bvsms.saude.gov.br/bvs/periodicos/ccs_artigos/v29_supl_alimentos_ultraprocessad os.p df. Aceso em 03 Jun.2020.

MATOS, H.C. et al. Tendência de consumo de alimentos industrializados por portadores de doenças crônicas não transmissíveis. Revista Contexto e Saúde vol.ro n.37; 2019. Disponível em: http://dx.doi.org/I0.21527/2176-7114.2019.37-37-43. Acesso em: I4 Maio de 2020. 
MINISTÉRIO DA SAÚDE (BRASIL). Instituto Nacional de Câncer José de Alencar Gomesda Silva. ABC do Câncer, Abordagens básicas para o controle do Câncer. $4^{\underline{a}}$ edição.Ministério da Saúde. Rio de Janeiro 2018. Disponível em;

https://www.inca.gov.br/sites/ufu.sti.inca.local/files/media/document/livro-abc-4edicao.pdf. Acesso em 2 Jun. 2020.

MINISTÉRIO DA SAÚDE (BRASIL). Secretaria de Atenção à Saúde. Guia Alimentar para a população brasileira. 2. ed. Brasília: Ministério da Saúde; 20r4. Disponível em: http://189.28.128.10o/dab/docs/portaldab/publicacoes/guia_alimentar_populacao_brasilei ra.pdf. Acesso em i4 Nov. 2019 e I4 Maio de 2020.

NEITZEL,B; et al. Fatores nutricionais associados a doenças crônicas não transmissíveis. Revista ciência em extensão v.I4, n.I, p.9-25,2018. Disponível em: https://ojs.unesp.br/index.php/revista_proex/article/view/r604. Acesso em: 02 Jun. 2020.

SOCIEDADE BRASILEIRA DE CARDIOLOGIA. $7^{\text {a }}$ Diretriz Brasileira de Hipertensão Arterial. Volume 107, № 3, Suplemento 3, Setembro 2016. Disponível em: http://publicacoes.cardiol.br/20I4/diretrizes/2016/o5_HIPERTENSAO_ARTERIAL.pdf.

Acesso em: 2 Jun. 2020.

SOCIE BRASILEIRA DE DIABETES. Diretrizes Sociedade Brasileira de Diabetes (20192020). Disponível em: https://www.diabetes.org.br/profissionais/images/DIRETRIZESCOMPLETA-2019-2020.pdf. Acesso em: 2 Jun. 2020. 